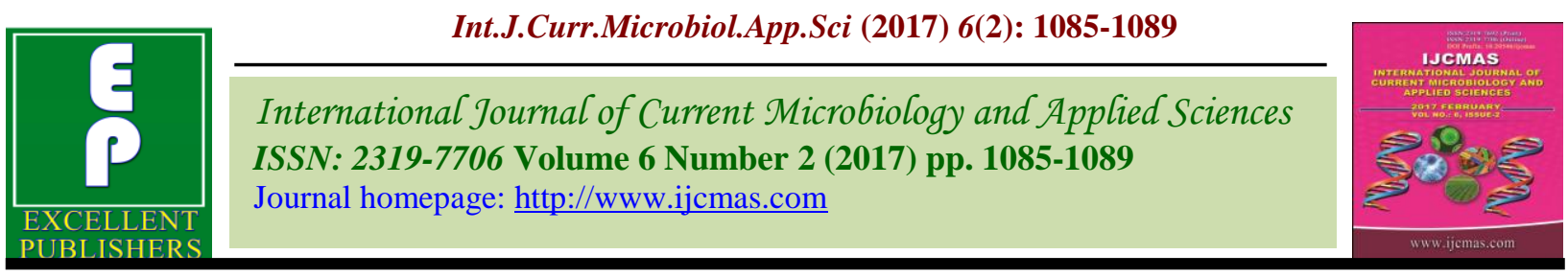

Original Research Article

http://dx.doi.org/10.20546/ijcmas.2017.602.122

\title{
Occurrence of Dengue Fever in Tertiary Care Hospitals
}

\author{
Arti Jain* \\ Sr.MIG, Block A - 16, Peoples College of Medical Sciences and Research Centre, PCMS \\ Campus, Bhanpur Road, Bhopal -462037, Madhya Pradesh, India \\ *Corresponding author
}

\begin{tabular}{|c|}
\hline Keywords \\
\hline $\begin{array}{l}\text { Dengue fever, } \\
\text { Mosquito-borne } \\
\text { disease, IgM, IgG } \\
\text { Antibodies and NS1 } \\
\text { Antigen. }\end{array}$ \\
\hline Article Info \\
\hline $\begin{array}{l}\text { Accepted: } \\
\text { 20 January } 2017 \\
\text { Available Online: } \\
\text { 10 February } 2017\end{array}$ \\
\hline
\end{tabular}

Keywords

Dengue fever, Mosquito-borne disease, IgM, IgG Antibodies and NS1

\section{A B S T R A C T}

Dengue is the most important mosquito-borne, human viral disease in many tropical and sub-tropical areas. India is endemic for Dengue infection. The purpose of this Study was to evaluate the occurrence of dengue fever among clinically suspected patients. We report a retrospective analysis of the dengue positivity in the serum samples tested during December, 2011 to November, 2012. This study was conducted in the Department of Microbiology, attached to a Tertiary Care Hospitals in Central India after obtaining ethical clearance over a time period of one year. A total of 731 serum samples on day one of fever from clinically Dengue suspected cases were received in the department of Microbiology. Of the 731 patients, 173 (23.6\%) were tested positive for Dengue infection. Among them, 26/173 (14.03\%) were from paediatric age while 147/173 $(84.97 \%)$ were adults. A total of 10 cases were positive for NS1 antigen and IgG antibodies while 6 cases tested positive for NS1 antigen and IgM antibody. A total of 110, 21 and 23 were positive for NS 1 antigen, IgG antibody and IgM antibody respectively. Remaining 3 were positive for $\mathrm{IgG}$ and $\mathrm{IgM}$ antibodies. Of these 173 positive cases, $110(63.5 \%)$ were primary infections and $63(36.5 \%)$ were secondary infections.

\section{Introduction}

Dengue fever and Dengue hemorrhagic fever (DHF) are acute febrile illnesses caused by four closely related virus serotypes of genus Flavivirus (DEN1-4). It is also known as break bone feve (World Health Organization, 2000). Dengue is transmitted to human by the bite of Aedes aegypti. Dengue is the most important mosquito-borne, human viral disease in many tropical and sub-tropical areas (Zafishan et al., 2008). Dengue is almost epidemic throughout in India for over two centuries as a benign and self-limited disease. In recent years, the disease has changed its course manifesting in the severe form as DHF and with increasing frequency of outbreaks (Gupta et al., 2006). The resource poor health system has to depend upon simple to perform and easy to interpret laboratory tests for diagnosis. It is known that early and specific diagnosis of DHF followed by supportive therapy reduces morbidity and mortality (Peters et al., 2008). The variability in the clinical illness associated with Dengue infection cannot be accommodated in a single clinical definition. However, confirmation of Dengue infection is the most essential pre- 
requisite in the management of complications. The 'gold standard test (viral isolation by cell culture) for identification of Dengue infection are not within the reach of peripheral and even most tertiary care laboratories. Detection of Dengue specific $\operatorname{IgM} / \mathrm{IgG}$ has been mainstay of diagnosis of Dengue infection. Antibody detection is an indirect method of diagnosis and therefore, is prone to false positive as well as false negative results (Peeling et al., 2010).

$\operatorname{IgM}$ antibody is the first immunoglobulin isotype to appear. In a suspected case of dengue, the presence of anti-dengue $\operatorname{IgM}$ antibody can detected usually fifth day of infection and suggests recent infection and a newer parameter NS1appears to be sensitive as well as highly specific and reliable for diagnosis of DI from the first day of fever.

We report a retrospective analysis of the dengue positivity in the serum samples tested during December, 2011 to November, 2012.

Aim of the study is to find the Occurrence of dengue fever among clinically suspected patients attending tertiary care hospital.

\section{Materials and Methods}

This Cross-sectional Analytical study was conducted in the Department of Microbiology, attached to a Tertiary Care Hospital in Central India from December, 2011 to November, 2012 after obtaining institutional Ethics Clearance.

\section{Collection of samples}

A total of 731 serum samples from suspected cases irrespective of age and sex were received in the department of Microbiology and transported immediately to the Microbiology laboratory for further processing. Of the 731 cases, 277 were females, 454 were males. 90 cases were from paediatric age and 641 were adults. The serum samples were tested for NS-1 antigen, IgG and IgM antibodies using rapid solid phase Immuno-chromatography test. (Dengue Day 1 test by J. Mitra and Co. Pvt. Ltd.). The tests were performed strictly as per the manufacturer's instruction. No other parameter included in our study.

\section{Results and Discussion}

Of the 731 patients, $173(23.6 \%)$ were tested positive. Among them, 26 (14.03\%) were from paediatric age (0-14 yrs) while 147 $(84.97 \%)$ were adults (>15 yrs) and out of 173 positive case 109 (63\%) were males, while females constituted only $64(37 \%)$. It clearly reveals that the highest number of cases belonged to the age group $\geq 15 \mathrm{yr}$ and males clearly outnumbered the females.

A total of 10 cases were positive for NS1 antigen and $\mathrm{IgG}$ antibodies while 6 cases were positive for NS1 antigen and IgM antibody. A total of 110, 21 and 23 were positive for NS 1 antigen, IgG antibody and $\operatorname{IgM}$ antibody respectively. Remaining 3 were positive for IgG and IgM antibodies (Table1).

The incidence of dengue has increased 30 fold with an expanded geographic distribution of both the virus and mosquito vector to new countries and from urban to rural settings (World Health Organization, 2009.).

It affects up to 100 million people each year, with 500,000 cases of dengue hemorrhagic fever (DHF) and dengue shock syndrome (DSS) and around 30,000 deaths, mostly amongst children. In view of the high mortality rate and to reduce the disease burden, it is imperative to have a rapid and sensitive laboratory assay for early detection of the disease. 
For a long time detection of dengue-specific $\mathrm{IgG} / \mathrm{IgM}$ antibodies has been the mainstay of diagnosis of dengue infection. These antibodies begin to appear only around fifth day of fever in primary infection. Even in most secondary infections, both IgM and IgG antibodies cannot be recorded before third day (Schilling et al., 2004). Therefore, there is always a window period, both in primary and secondary dengue infection when only antibodies are tested.

The new parameter, NS1 antigen, is detectable from day 1 of fever both in primary and secondary infections making it highly specific parameter for the diagnosis from day 1 of the fever.

In this study $23.5 \%$ cases were serologically positive for dengue infection. Similar findings were reported by Garg et al., (2011); Balaya et al., (1967); Gupta et al., (2012).

The distribution of various dengue specific parameters with other studies is shown in (Table 2). In this study, of the 173 cases, 110 $(63.5 \%)$ were positive for NS1 antigen only. This finding is consistent with the study done by Kulkarni et al., (2011), Saurabh Agarwal et al., (2014) and Parameswarappa Jyothi et al., (2015) in which 30\%, 29.4\% and $62.6 \%$ cases were positive for only NS1 antigen respectively. The positivity with NS1 is higher than other parameters because NS1 appears to be highly specific and reliable for diagnosis of dengue infection from the first day of fever.

Table.1 Comparison of various dengue specific parameters in diagnosing dengue infection

\begin{tabular}{|c|c|c|}
\hline Parameters & Numbers & Percentage (\%) \\
\hline NS1 only & 110 & 63.2 \\
\hline IgM only & 23 & 13.2 \\
\hline IgG only & 21 & 12.1 \\
\hline NS1+IgM & 06 & 3.4 \\
\hline NS1+IgG & 10 & 5.7 \\
\hline IgM+IgG & 03 & 1.7 \\
\hline Total & $\mathbf{1 7 3}$ & $\mathbf{1 0 0 \%}$ \\
\hline
\end{tabular}

Table. 2 Comparison of various dengue specific parameters with other studies in diagnosing dengue infection

\begin{tabular}{|l|c|c|c|c|c|c|}
\hline \multirow{2}{*}{\multicolumn{1}{c|}{ Authors }} & \multicolumn{7}{c|}{ Specific parameters } \\
\cline { 2 - 7 } & NS1 only & IgM only & IgG only & NS1+IgM & NS1+IgG & IgM+IgG \\
\hline Kulkarni RD et al $^{15}$ & $30 \%$ & $50 \%$ & $3 \%$ & $11 \%$ & $0.3 \%$ & $6 \%$ \\
\hline Saurabh G Agarwal et $_{\text {al }} \mathbf{l}^{16}$ & $29.4 \%$ & $35.2 \%$ & $05.88 \%$ & $05.88 \%$ & $05.88 \%$ & $23.52 \%$ \\
\hline $\begin{array}{l}\text { Parameswarappa Jyothi } \text { et } \\
\text { al }^{17}\end{array}$ & $62.9 \%$ & $11.3 \%$ & $4.9 \%$ & $9.6 \%$ & $1.7 \%$ & $9.6 \%$ \\
\hline Saroj Golia at al $^{18}$ & 23.3 & 33.3 & 16.7 & 12.2 & 1.1 & 12.2 \\
\hline \multicolumn{1}{|c|}{ Our Study } & $\mathbf{6 3 . 5 \%}$ & $\mathbf{1 3 . 2 \%}$ & $\mathbf{1 2 . 1 \%}$ & $\mathbf{3 . 4 \%}$ & $\mathbf{5 . 7 \%}$ & $\mathbf{1 . 7 \%}$ \\
\hline
\end{tabular}

IgM only was positive in $23(13.2 \%)$ cases. Other study conducted by Saurabh et al., and Kulkarni et al., shows $35.2 \%$ and $50 \%$ patients serologically positive for dengue infection respectively (Table 2). This indicates that, dengue-specific $\operatorname{IgM}$ is a very good indicator of recent infection. 
IgG was positive only in $3 \%$ cases which showed past infection with dengue. Both IgM and $\mathrm{IgG}$ were found to be positive for $1.7 \%$ cases indicating acute or chronic infection. Similar findings showed by Kulkarni et al., (2011) and Parameswarappa Jyothi et al., (2015) (Table 3).

NS1 in combination with either $\operatorname{IgM}$ or $\operatorname{IgG}$ was positive in 16 (NS1+IgM=06, $\mathrm{NS} 1+\mathrm{IgG}=10$ ) cases in my study. Among these two antibodies, IgG is a less reliable marker. Both clinical and sub-clinical infections can produce IgG which may persist for several years affecting the interpretation of test results. It is highly likely that $\mathrm{IgG}$ levels could be higher in endemic areas because of bites from infected mosquitoes.

A gradual increase of cases was noticed from August with a peak in October 2012 which correlates with occurrence of Dengue with monsoon season. It is supported by similar finding (Gunasekaran et al., 2011). It is because of mosquitoes breeding more in August- October monsoon season.

A higher prevalence of dengue infection was noted among males. The male to female ratio was 2:1 which is in congruent with other studies. The male predominance is may be due to higher level of outdoor activity.

Primary infection was noted in $63.5 \%$ cases while secondary infection in $36.5 \%$ cases (Table1).

This study has been carried out at a tertiary care hospital. It is worth mentioning here that most tertiary care hospitals lack in viral culture setup. Therefore, applying gold standard tests in studies related to viral infections is out of reach of these centers.

The limitation of the present study was that enzyme linked immunosorbent assay (ELISA) for qualitative or quantitative detection or
Polymerase Chain Reaction (PCR) could not be used.

In conclusion, occurrence of dengue infection in tertiary care hospital for period December 2011-November 2012 is 23.1\%. Maximum cases were reported during SeptemberOctober, 2012.

NS1 testing must be included for evaluation of all cases of fever, either in endemic or nonendemic areas during this period, to rule out acute dengue infection.

\section{References}

Atul Garg, Jaya Garg, Y.K. Rao, G.C. Upadhyay, Suman Sakhuja. 2011. Prevalance of dengue among clinically suspected febrile episodes at a teaching hospital in North India. JIDM, Vol. 3(5), PP.85-89.

Balaya, S., Paul, S.D., D’Lima, L.V., Pavri, K.M. 1967. Investigation on an outbreak of Dengue in Delhi in IJMR, 57: 767-775.

Datta, S., Wattal, C. 2010. Dengue NS1 antigen detection: A useful tool in early diagnosis of dengue virus infection. Indian J. Med. Microbiol., 28: 107.

Gunasekaran, K., Kaveri, S., Mohana, K., Arunagiri, B.V., Suresh Babu, R. Kiruba. 2011. Dengue disease status in Chennai (2006-2008), A retrospective analysis. IJMR, 133-325.

Gupta, E., L. Dar, G. Kapoor, and S. Broor. 2006. "The changing epidemiology of dengue in Delhi, India," Virol. J., vol. 3, article 92, 2006.

Guzman, M.G., Kouri, G. 2002. Dengue: an update. Lancet Infect. Dis., 2: 33-42.

Kulkarni, R.D., S.S. Patil, R.D. Kulkarni, S.S. Patil, G.S. Aja. 2011. Association of platelet count and serological markers of dengue infection importance of 
NS1 antigen. Indian J. Med. Microbiol., 29(4): 359-62.

Libraty, D.H., Young, P.R., Pickering, D., Endy, T.P., Kalayanarooj, S., Green, S., et al. 2002. High Circulating Levels of the Dengue Virus Nonstructural Protein NS1 Early in Dengue Illness Correlate with the Development of Dengue Hemorrhagic Fever. J. Infect. Dis., 186:1165-8.

Nivedita Gupta, Amita Jain. 2012. Dengue in India, IJMR, 136, p 373-390.

Parameswarappa Jyothi, Basavaraj, C., Metri, et al. 2015. Correlation of serological markers and platelet count in the diagnosis of Dengue virus infection. Adv. Biomed. Res., 4: 26.

Peeling, R.W., Artsob, H., Pelegrino, J.L., Buchy, P., Cardosa, M.J., Devi, S., et al.2010. Evaluation of diagnostic tests: Dengue. Nat. Rev. Microbiol., 8: S30-7.

Peters, C.J. 2008. Infection caused by arthropod and Rodent born viruses, In; Fauci as editor. Harisson's principle of internal medicine.17th ed. New York: Mc Graw-Hill Medical Publishing Division, p.1226-39.

Saroj Golia, Saroj Golia Vivek Hittinahalli. 2012. Serodiagnosis of Dengue using NS1 antigen, dengue IgM, dengue $\mathrm{IgG}$ antibodies with correlation of platelet counts. IJ-AJIMS, 1: 112-117.

Saurabh, G., Agarwal. 2014. "Importance of Platelet Count and Serological
Markers in Diagnosing Dengue Infection with Special Reference to NS1 Antigen". J. Evol. Med. Dental Sci., Vol. 3, Issue 10, March 10; Page: 2543-2547.

Schilling, S., Ludolfs, D., An, L.V., Schmitz, H. 2004. Laboratory diagnosis of primary and secondary dengue infection. J. Clin. Virol., 31: 179-84.

Shrivastava, A., Dash, P.K., Tripathi, N.K., Sahni, A.K., Gopalan, N., Lakshmana, Rao, P.V. 2011. Evaluation of a commercial dengue NS1 enzyme-linked immunosorbent assay for early diagnosis of dengue infection. Indian J. Med. Microbiol., 29: 51-5.

World Health Organization, 2009. Dengue, guidelines for diagnosis, treatment, prevention and control.

World Health Organization. 1997. Dengue haemorrhagic fever: Diagnosis, treatment, prevention and control (second edition). Geneva, Switzerland: Chapter 4, Laboratory diagnosis, $\mathrm{p}$. 34-47.

World Health Organization. 2000. Scientific Working Grow-up on Dengue. Meeting report, Geneva, Switzerland, Geneva: WHO, 2000.

Zafishan Tahir, Salma Hafeez, Asalm Chaudhary. 2008. Spatial and seasonal variation of dengue fever in Lahore, Biomedica, Vol. 26, P.166-172.

\section{How to cite this article:}

Arti Jain. 2017. Occurrence of Dengue Fever in Tertiary Care Hospitals. Int.J.Curr.Microbiol.App.Sci. 6(2): 1085-1089. doi: http://dx.doi.org/10.20546/ijcmas.2017.602.122 